УДК 519.224

\title{
О СОВМЕСТНОМ РАСПРЕДЕЛЕНИИ ЭКСТРЕМАЛЬНЫХ СТЬЮДЕНТИЗИРОВАННЫХ ОТКЛОНЕНИЙ, ВЫЧИСЛЕННЫХ ПО НОРМАЛЬНОЙ ВЫБОРКЕ ИЗ ТРЕХ НАБЛЮДЕНИЙ
}

\section{Ширяева Людмила Константиновна}

к.ф.-М. н., доцент

ФГАОУ ВО "Самарский государственный экономический университет"

Аннотация: Рассмотрены экстремальные стьюдентизированные отклонения наблюдений нормальной выборки от среднего. Показано, что в случае трех наблюдений функция совместного распределения этих экстремальных отклонений может быть задана в явном виде. Разработан алгоритм построения графика этой функции в среде MAPLE.

Ключевые слова: экстремальные стьюдентизированные отклонения наблюдений от среднего, нормальный закон распределения, совместная функция распределения, рекурсия, среда программирования MAPLE.

\section{ON THE JOINT DISTRIBUTION OF EXTREME STUDENTIZED DEVIATIONS CALCULATED FROM A NORMAL SAMPLE OF THREE OBSERVATIONS}

\section{Shiryaeva Lyudmila Konstantinovna}

Abstract: Extreme studentized deviations of observations of a normal sample from the average are considered. It is shown that in the case of three observations, the function of the joint distribution of these extreme deviations can be given explicitly. An algorithm for plotting this function in the MAPLE environment has been developed.

Key words: extreme studentized deviations of observations from the mean, normal distribution, joint distribution function, recursion, MAPLE programming environment.

Копула-функции часто применяют для решения задач финансовой и страховой математики. Одним из источников копул являются совместные 246 
распределения случайных величин [1, с.51]. Например, из совместного нормального распределения была построена копула Гаусса, а из совместного $t$ распределения - $t$-копула Стьюдента. Любое новое совместное распределение случайных величин можно рассматривать как потенциальный источник новой копулы. Поэтому исследование новых совместных распределений случайных переменных представляется актуальной задачей. В данной работе рассмотрены односторонние статистики Граббса [2] и исследовано их совместное распределение, порожденное тремя наблюдениями.

Пусть $X_{1}, X_{2}, \cdots, X_{n}$ - случайная выборка из $n$ значений нормально распределенной случайной величины $X$. Построим по этой выборке вариационный ряд $X_{(1)} \leq X_{(2)} \leq \cdots \leq X_{(n-1)} \leq X_{(n)}$ и рассмотрим случайные величины, являющиеся экстремальными стьдентизированными отклонениями наблюдений от среднего по выборке, т.е.

$$
T_{n}^{(1)}=\frac{X_{(n)}-\bar{X}}{S} ; T_{n,(1)}=\frac{\bar{X}-X_{(1)}}{S},
$$

где $\bar{X}=\frac{1}{n} \sum_{i=1}^{n} X_{i}, S=\sqrt{\frac{1}{n-1} \sum_{i=1}^{n}\left(X_{i}-\bar{X}\right)^{2}}$.

Легко убедиться, что $P\left(T_{n,(1)}<t\right)=P\left(T_{n}^{(1)}<t\right)$. Обозначим

$$
F_{n}^{(1)}(t)=P\left(T_{n}^{(1)}<t\right) \text {. }
$$

Рекурсивные формулы, описывающие функцию распределения случайной величины $T_{n}^{(1)}$, имеют вид [3]

$$
F_{n}^{(1)}(\mathrm{t})=\left\{\begin{array}{l}
0, \quad t \leq \frac{1}{\sqrt{n}}, n \geq 2 \\
n \int_{1 / \sqrt{n}}^{t} F_{n-1}^{(1)}\left(g_{n}(x)\right) f_{T_{n}}(x) d x, \quad \frac{1}{\sqrt{n}}<t \leq \frac{n-1}{\sqrt{n}}, n>2 \\
1, \quad t>\frac{n-1}{\sqrt{n}}, n \geq 2
\end{array}\right.
$$

где $g_{n}(t)=\frac{n t}{n-1} / \sqrt{\frac{n-1}{n-2}\left(1-\frac{n t^{2}}{(n-1)^{2}}\right)}$;

$f_{T_{n}}(x)=\frac{\sqrt{n}}{(n-1) \sqrt{\pi}} \cdot \frac{\Gamma((n-1) / 2)}{\Gamma((n-2) / 2)} \cdot\left(1-\frac{n x^{2}}{(n-1)^{2}}\right)^{\frac{n-4}{2}} ; \Gamma(x)$-гамма-функция.

Обозначим: $\Lambda_{n}\left(t_{1}, t_{2}\right)=P\left(T_{n,(1)}<t_{1}, T_{n}^{(1)}<t_{2}\right)$. 
В [4] найдено, что рекурсивные формулы для совместной функции распределения $\Lambda_{n}\left(t_{1}, t_{2}\right)$ для случая $n>2$ имеют вид:

$$
\begin{aligned}
& \Lambda_{n}\left(t_{1}, t_{2}\right)= \begin{cases}F_{n}^{(1)}\left(t_{2}\right), \quad t_{1} \geq \frac{n-1}{\sqrt{n}}, & n>2 ; \\
F_{n}^{(1)}\left(t_{1}\right), \quad t_{2} \geq \frac{n-1}{\sqrt{n}}, & n>2 ; \\
n \int_{1 / \sqrt{n}}^{t_{2}} \Lambda_{n-1}\left(\rho_{n}\left(t_{1},-x\right), g_{n}(x)\right) f_{T_{n}}(x) d x, n>2, \quad\left(t_{1}, t_{2}\right) \in \Delta_{n} ; & \\
0, \quad\left(t_{1}, t_{2}\right) \notin \Delta_{n}, t_{1}<\frac{n-1}{\sqrt{n}} ; t_{2}<\frac{n-1}{\sqrt{n}}, \quad n>2,\end{cases} \\
& \text { где } \rho_{n}(t, z)=\frac{t+\frac{z}{n-1}}{\sqrt{\frac{n-1}{n-2}\left(1-\frac{n z^{2}}{(n-1)^{2}}\right)}} ; \quad \Delta_{n}=\left[\frac{1}{\sqrt{n}}, \frac{n-1}{\sqrt{n}}\right]^{2}, n>2 .
\end{aligned}
$$

В случае $n=2$ имеем [4]

$$
\Lambda_{2}\left(t_{1}, t_{2}\right)=\left\{\begin{array}{l}
0, \text { если }\left(t_{1}, t_{2}\right) \notin \Delta_{2} ; \\
1, \text { если }\left(t_{1}, t_{2}\right) \in \Delta_{2},
\end{array} \quad \Delta_{2}=\left[\frac{1}{\sqrt{2}}, \infty\right]^{2} .\right.
$$

Из соотношений (1) и (2) вытекает, что для $n=2$ совместная функции распределения случайных величин $T_{n}^{(1)}$ и $T_{n,(1)}$ задана явным образом, а при $n \geq 3$ вычисление ее значений уже требует применения рекурсивных алгоритмов. Однако с точки зрения вычислительной реализации при $n>2$ такие рекурсивные алгоритмы могут быть крайне ресурсоемкими.

В данной работе мы покажем, что для $n=3$ рекурсивные соотношения (2) могут быть приведены к соотношениям нерекурсивного вида. Для этого заметим, что при $n=3$ маргинальная функция распределения случайной величины $T_{3}^{(1)}$ описывается следующим образом [4]

$$
F_{3}^{(1)}(\mathrm{t})=\left\{\begin{array}{l}
0, \quad t \leq \frac{1}{\sqrt{3}} \\
\frac{3}{\pi} \arcsin \left(\frac{\sqrt{3}}{2} t\right)-\frac{1}{2}, \quad \frac{1}{\sqrt{3}}<t \leq \frac{2}{\sqrt{3}} \\
1, \quad t>\frac{2}{\sqrt{3}}
\end{array}\right.
$$

Кроме того, в случае $n=3$ совместная функция распределения случайных величин $T_{3}^{(1)}$ и $T_{3,(1)}$ имеет вид [5] 


$$
\Lambda_{3}\left(t_{1}, t_{2}\right)=\left\{\begin{array}{l}
F_{3}^{(1)}\left(t_{2}\right), \quad t_{1} \geq \frac{2}{\sqrt{3}} \\
F_{3}^{(1)}\left(t_{1}\right), \quad t_{2} \geq \frac{2}{\sqrt{3}} \\
F_{3}^{(1)}\left(t_{1}\right)+F_{3}^{(1)}\left(t_{2}\right)-1, \quad\left(t_{1}, t_{2}\right) \in \Sigma_{3} \\
0, \quad\left(t_{1}, t_{2}\right) \in \Delta_{3} \backslash \Sigma_{3},
\end{array}\right.
$$

где $\Sigma_{3}=\left[\frac{1}{\sqrt{3}} \leq t_{1} \leq \frac{2}{\sqrt{3}} ; \theta_{3}\left(t_{1}\right) \leq t_{2} \leq \frac{2}{\sqrt{3}}\right] ; \quad \theta_{3}\left(t_{1}\right)=\frac{t_{1}}{2}+\sqrt{1-\frac{3}{4} t_{1}^{2}}$.

Тогда с учетом (3) и (4) получим

$$
\Lambda_{3}\left(t_{1}, t_{2}\right)=\left\{\begin{array}{l}
0, \quad\left(t_{1}, t_{2}\right) \in \Delta_{3} \backslash \Sigma_{3} \\
\frac{3}{\pi} \arcsin \left(\frac{\sqrt{3}}{2} t_{1}\right)+\frac{3}{\pi} \arcsin \left(\frac{\sqrt{3}}{2} t_{2}\right)-2, \quad\left(t_{1}, t_{2}\right) \in \Sigma_{3}
\end{array}\right.
$$

На рисунке 1 приведен график функции $\Lambda_{3}\left(t_{1}, t_{2}\right)$ для $\left(t_{1}, t_{2}\right) \in \Delta_{3}$. График построен с помощью пакета аналитических вычислений MAPLE. Для рисования графика по точкам $\left(t_{1}, t_{2}, \Lambda_{3}\left(t_{1}, t_{2}\right)\right)$ применялась процедура pointplot3d из пакета plots.

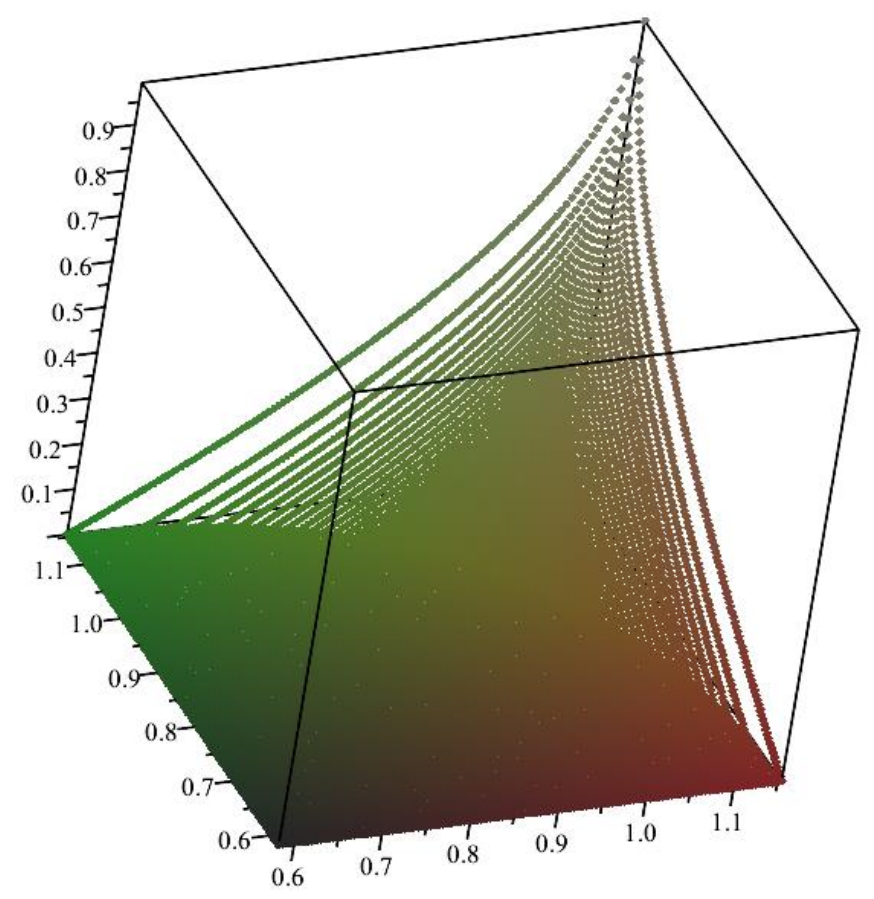

Рис. 1. График функции $\Lambda_{3}\left(t_{1}, t_{2}\right)$ для $\left(t_{1}, t_{2}\right) \in \Delta_{3}$ 


\section{Список литературы}

1. Nelsen R.B. An Introduction to Copulas. Lecture Notes in Statistics. - New York: Springer-Verlag, -2006 . -276 p.

2. Grubbs F. Sample criteria for testing outlying observations// Ann. Math. Statist. - 1950. -V. 21(1), p. 27-58.

3. Zhang J., Keming Y. The null distribution of the likelihood-ratio test for one or two outliers in a normal sample// TEST. - 2006. - V.15 (1), p.141-150.

4. Ширяева Л.К. О нулевом и альтернативном распределении статистики критерия наибольшего по абсолютной величине нормированного отклонения//Изв. вузов. Математика. - 2014. - № 10. - С. $62-78$.

5. Ширяева Л.К. О трехпараметрической копула-функции Граббса //Изв. вузов. Математика. - 2019. - № 3. - С. 54-71.

() Л.К. Ширяева, 2021 